\title{
Variational Iteration Method for Nonlinear Singular Two-Point Boundary Value Problems Arising in Human Physiology
}

\author{
Marwan Abukhaled \\ Department of Mathematics and Statistics, American University of Sharjah, P.O. Box 26666, Sharjah, UAE \\ Correspondence should be addressed to Marwan Abukhaled; mabukhaled@aus.edu
}

Received 15 November 2012; Accepted 13 December 2012

Academic Editor: Jen-Chih Yao

Copyright (C) 2013 Marwan Abukhaled. This is an open access article distributed under the Creative Commons Attribution License, which permits unrestricted use, distribution, and reproduction in any medium, provided the original work is properly cited.

\begin{abstract}
The variational iteration method is applied to solve a class of nonlinear singular boundary value problems that arise in physiology. The process of the method, which produces solutions in terms of convergent series, is explained. The Lagrange multipliers needed to construct the correctional functional are found in terms of the exponential integral and Whittaker functions. The method easily overcomes the obstacle of singularities. Examples will be presented to test the method and compare it to other existing methods in order to confirm fast convergence and significant accuracy.
\end{abstract}

\section{Introduction}

In this paper, He's variational iteration method (VIM) [1, 2] is applied to obtain an approximate solution for the following nonlinear singular two-point boundary value problem (BVP):

$$
\left(p(x) y^{\prime}\right)^{\prime}=p(x) f(x, y), \quad 0 \leq x \leq 1,
$$

with the following two sets of boundary conditions:

$$
\begin{array}{ll}
y^{\prime}(0)=0, & \alpha y(1)+\beta y^{\prime}(1)=\gamma, \\
y(0)=A, & \alpha y(1)+\beta y^{\prime}(1)=\gamma,
\end{array}
$$

where

$$
p(x)=x^{b} h(x), \quad x \in[0,1] .
$$

Here $\alpha>0, \beta \geq 0$, and $A$ and $\gamma$ are finite constants. It is assumed that $p(x)$ is nonnegative, continuously differentiable on $[0,1]$, and $1 / h(x)$ is analytic in the disk $|z|<r$ for some $r>1$. It is also assumed that $f(x, y)$ and $\partial f / \partial y$ are both continuous on $[0,1] \times \mathbb{R}$ and further $\partial f / \partial y \geq 0$.

Assuming that $x p^{\prime} / p$ is analytic in $|z|<r$ for some $r>1$, the existence-uniqueness has been established for problem (1) under the following restrictions (see [3] and the references therein):

(1) BC (2) holds for $\alpha=1, \beta=0$ and such that $b \geq 0$, and

(2) $\mathrm{BC}$ (3) holds for $\alpha=1, \beta=0$, and such that $0 \leq b \leq 1$.

Equation (1) with BC (3) arises in the study of tumor growth problems $[4,5]$ where $b=0,1,2, h(x)=1$, and $f(x, y)$ is of the form

$$
f(x, y)=\frac{\theta y}{y+\kappa}, \quad \theta, \kappa>0 .
$$

This problem also arises in the study of a steady-state oxygen diffusion in a cell with Michaleis-Menten uptake kinetics when $b=2$ and $h(x)=1$ [6]. Another application of this problem appeared in the study of heat sources distribution in the human head [7] given that $b=2, h(x)=1$, and

$$
f(x, y)=-\delta e^{-\theta y}, \quad \theta, \delta>0 .
$$

Problems (1)-(2) and (1)-(3) have been treated by several authors using different numerical schemes such as cubic Bsplines, finite difference, and pointwise solution bounds [3, $8,9]$. The VIM has been used by Wazwaz [10] to solve the nonlinear singular Emden-Fowler boundary value problem, which is a special case of (1)-(2), where $p(x)=x^{b}$. Also, Ravi 
Kanth and Aruna [11] used the variational iteration method to solve (1)-(2) and (1)-(3), but that was also done under the assumption that $h(x)=1$.

The objective of this paper is to apply the VIM to obtain an approximate solution for the proposed nonlinear singular boundary value problems with more relaxed $p(x)$. The VIM has been intensively used to solve linear and nonlinear, ordinary and partial, delay and fractional order differential equations [12-18]. In a recent review article, $\mathrm{He}$ [19] shows how the VIM can be employed as an effective method in searching for wave solutions including solitons and compacton solutions without the need for linearization or weak nonlinearity assumptions. For nonlinear differential equations, the VIM gives an approximate solution in a series form that converges to the exact solution, if the latter exists. In case of linear differential equations, exact solution by the VIM can be readily obtained by a one iteration step because the exact Lagrange multiplier needed to construct the correctional functional can be identified.

\section{Variational Iteration Method}

Consider the general nonlinear differential equation given by

$$
L y(t)+N y(t)=g(t),
$$

where $L$ and $N$ are linear and nonlinear operators, respectively, and $g(t)$ is a given analytical function. As per the variational iteration method, the correction functional is constructed as follows:

$$
\begin{aligned}
y_{n+1}(x)= & y_{n}(x) \\
& +\int_{0}^{x} \lambda(\xi)\left(L y_{n}(\xi)+N y_{n}(\xi)-g(\xi)\right) d \xi
\end{aligned}
$$

where $\lambda$ is a general Lagrange multiplier, which can be optimally identified by the variational theory, and $\widetilde{y}_{n}$ is a restricted variation and hence $\delta \widetilde{y}_{n}=0$. A proper choice of the initial approximation $y_{0}$ is crucial to obtain a successive approximations $y_{n+1}$ that converge as rapidly as possible to the exact solution.

For simplicity, (1) will be expressed in the form

$$
y^{\prime \prime}+\left(\frac{b}{x}+\frac{h^{\prime}(x)}{h(x)}\right) y^{\prime}=f(x, y)
$$

and its correction functional is given by

$$
\begin{aligned}
y_{n+1}(x)= & y_{n}(x)+\int_{0}^{x} \lambda(\xi) \\
& \times\left(\left(y_{n}(\xi)\right)_{\xi \xi}+\left(\frac{b}{\xi}+\frac{h^{\prime}(\xi)}{h(\xi)}\right)\left(y_{n}(\xi)\right)_{\xi}\right. \\
& \left.-\tilde{f}\left(\xi, y_{n}\right)-g(\xi)\right) d \xi .
\end{aligned}
$$

Taking the variation with respect to $y_{n}$ gives

$$
\begin{aligned}
\delta y_{n+1}(x)= & \delta y_{n}(x)+\delta \int_{0}^{x} \lambda(\xi) \\
& \times\left(\left(y_{n}(\xi)\right)_{\xi \xi}+\left(\frac{b}{\xi}+\frac{h^{\prime}(\xi)}{h(\xi)}\right)\left(y_{n}(\xi)\right)_{\xi}\right) d \xi
\end{aligned}
$$

Using integration by parts in (11) gives

$$
\begin{aligned}
& \delta y_{n+1}(x) \\
& =\delta y_{n}(x)\left(1-\lambda^{\prime}(x)+\left(\frac{b}{x}+\frac{h^{\prime}(\xi)}{h(\xi)}\right) \lambda(x)\right) \\
& +\delta\left(y_{n}\right)_{\xi}(x) \lambda(x) \\
& +\int_{0}^{x} \delta y_{n}(\xi)\left(\lambda^{\prime \prime}(\xi)-\left(\frac{b}{\xi}+\frac{h^{\prime}(\xi)}{h(\xi)}\right) \lambda^{\prime}(\xi)\right. \\
& \left.+\frac{b}{\xi^{2}} \lambda(\xi)\right) d \xi,
\end{aligned}
$$

which in turn leads to the stationary relations

$$
\begin{gathered}
1-\lambda^{\prime}(x)+\left(\frac{b}{x}+\frac{h^{\prime}(x)}{h(x)}\right) \lambda(x)=0, \\
\lambda(x)=0, \\
\lambda^{\prime \prime}(x)-\left(\frac{b}{x}+\frac{h^{\prime}(x)}{h(x)}\right) \lambda^{\prime}(x)+\frac{b}{x^{2}} \lambda(x)=0 .
\end{gathered}
$$

Depending on $b$, the Lagrange multiplier can be determined by solving system (13). In the next section, we will use $h(x)=e^{x}$, and hence the choice of $b$ will lead to one of the following two Lagrange multipliers.

If $b \geq 1$, the solution of (13) is given in terms of the exponential integral $E_{n}(x)$ [20]. For example, if $b=1$, then

$$
\lambda(\xi)=\left(E_{1}(\xi)-E_{1}(x)\right) \xi e^{\xi}
$$

where $E_{1}(x)$ is the generalized complex exponential integral given by

$$
E_{1}(x)=\int_{x}^{\infty} \frac{e^{-t}}{t} d t
$$

If $b<1$, the solution of (13) is given in terms of Whittaker function $M_{p, q}(x)$ [20]. For example, if $b=1 / 4$, then

$$
\begin{aligned}
\lambda(\xi)=\frac{4 e^{-x}}{21 x^{5 / 8} \xi^{3 / 8}}( & -\alpha(x, \xi) M_{3 / 8,7 / 8}(x)-\beta(x, \xi) \\
& \left.+\beta(\xi, x)+\alpha(\xi, x) M_{3 / 8,7 / 8}(\xi)\right),
\end{aligned}
$$


where

$$
\begin{gathered}
\alpha(m, n)=4 m n^{5 / 8} e^{1 / 2 m+n}, \\
\beta(m, n)=7 m^{11 / 8} n^{5 / 8} e^{n},
\end{gathered}
$$

and $M_{p, q}(x)$ is given by

$$
M_{p, q}(x)=e^{-x / 2} x^{q+1 / 2} \sum_{k=0}^{\infty} \frac{(1 / 2+q-p)_{k}}{k !(2 q+1)_{k}} x^{k},
$$

in which $(a)_{n}$ is known as the Pochhammer symbol [20] and is defined by

$$
\begin{gathered}
(a)_{n}=a(a+1)(a+2) \cdots(a+n-1), \\
(a)_{0}=1 .
\end{gathered}
$$

Now that $\lambda$ is constructed, an appropriate first guess $y_{0}(x)$ will lead to the formation of the recurrence sequences $\left\{y_{n}(x)\right\}$. The solution for (1)-(2) and (1)-(3) is, then, obtained from the relation

$$
y(x)=\lim _{n \rightarrow \infty} y_{n}(x),
$$

provided that the limit exists. The proof that the sequences $\left\{y_{n}(x)\right\}$ are convergent has been well established in the literature (see, e.g., $[11,21]$ ).

\section{Numerical Examples}

Example 1. Consider the nonlinear singular BVP

$$
\begin{aligned}
& \left(p(x) y^{\prime}\right)^{\prime}=p(x) f(x, y), \quad x \in[0,1], \\
& y^{\prime}(0)=0, \quad y(1)+5 y^{\prime}(1)=-5-\ln 5,
\end{aligned}
$$

where

$$
\begin{gathered}
p(x)=x^{b} g(x), \quad g(x)=e^{x}, \\
f(x, y)=\frac{5 x^{3}\left(5 x^{5} e^{y}-x-b-4\right)}{4+x^{5}} .
\end{gathered}
$$

The analytic solution of (21) is $y=-\ln \left(x^{5}+4\right)$ for all $b \in R$. This problem is an application of oxygen diffusion corresponding to (1), (2), and (5) with $p(x)=x^{2}, \theta=$ $0.76129, \kappa=0.03119, \alpha=5, \beta=1$, and $\gamma=5$. In Tables 1 and 3 , the second and third iteration solutions are compared to the exact solution when $b=0.25$ and $b=8$, respectively. In Tables 2 and 4, the absolute maximum errors obtained by the proposed VIM is compared with that of other methods.

Example 2. Consider the nonlinear singular BVP

$$
\begin{gathered}
\left(p(x) y^{\prime}\right)^{\prime}=p(x) f(x, y), \quad x \in[0,1], \\
y(0)=-\ln 4, \quad y(1)+5 y^{\prime}(1)=-5-\ln 5,
\end{gathered}
$$

where $p(x), g(x)$, and $f(x, y)$ are as in (22).
TABLE 1: Numerical results for Example $1(b=0.25)$.

\begin{tabular}{lccc}
\hline$x$ & $y_{2}$ solution & $y_{3}$ solution & Exact solution \\
\hline 0.1 & -1.386471621 & -1.386300039 & -1.386296861 \\
0.2 & -1.386549118 & -1.386377536 & -1.386374358 \\
0.3 & -1.387076437 & -1.386904854 & -1.386901677 \\
0.4 & -1.389025850 & -1.388854268 & -1.388851090 \\
0.5 & -1.394251264 & -1.394079679 & -1.394076502 \\
0.6 & -1.405722595 & -1.405550997 & -1.405547818 \\
0.7 & -1.427627934 & -1.427456278 & -1.427453099 \\
0.8 & -1.465206626 & -1.465034787 & -1.465031602 \\
0.9 & -1.524162176 & -1.523989964 & -1.523986772 \\
1.0 & -1.609612516 & -1.609441112 & -1.609437912 \\
\hline
\end{tabular}

TABle 2: Maximum error for Example $1(b=0.25)$.

\begin{tabular}{lcc}
\hline Present method & Method in [9] & Method in [3] \\
\hline $1.74 \times 10^{-4}(n=2)$ & $7.79 \times 10^{-4}(n=16)$ & $1.17 \times 10^{-3}(n=16)$ \\
$3.20 \times 10^{-6}(n=3)$ & $1.98 \times 10^{-4}(n=32)$ & $3.04 \times 10^{-4}(n=32)$ \\
\hline
\end{tabular}

TABle 3: Numerical results for Example $1(b=8)$.

\begin{tabular}{lccc}
\hline$x$ & $y_{2}$ solution & $y_{3}$ solution & Exact solution \\
\hline 0.1 & -1.386409538 & -1.386300604 & -1.386296861 \\
0.2 & -1.386487035 & -1.386378101 & -1.386374358 \\
0.3 & -1.387014353 & -1.386905420 & -1.386901677 \\
0.4 & -1.388963767 & -1.388854833 & -1.388851090 \\
0.5 & -1.394189179 & -1.394080244 & -1.394076502 \\
0.6 & -1.405660501 & -1.405551561 & -1.405547818 \\
0.7 & -1.427565803 & -1.427456843 & -1.427453099 \\
0.8 & -1.465144370 & -1.465035348 & -1.465031602 \\
0.9 & -1.524099638 & -1.523990525 & -1.523986772 \\
1.0 & -1.609550088 & -1.609441663 & -1.609437912 \\
\hline
\end{tabular}

This problem is an application of the nonlinear heat conduction model of the human head corresponding to (1), (3), and (6) with $p(x)=x^{2}, \delta=\theta=1$, and $\gamma=0$. In Tables 5 and 7 , the second and third iteration solutions are compared to the exact solution when $b=0.25$ and $b=0.75$, respectively, and in Tables 6 and 8, the absolute maximum errors obtained by the proposed VIM is compared with that of other methods.

\section{Conclusion}

In this paper, the simplicity and reliability of He's variational iteration method have been tested against a general form of nonlinear two point singular value problems that arise in physiology. The method is shown to be highly accurate and easily implemented even with the presence of singularities. For the two examples shown in Section 3, the second and third iteration solutions obtained by the VIM were substantially more accurate than the 16 and the 32 time-step approximate solutions obtained by cubic B-spline and the finite difference methods. 
TABle 4: Maximum error for Example $1(b=8)$.

\begin{tabular}{lcc}
\hline Present method & Method in [9] & Method in [3] \\
\hline $1.12 \times 10^{-4}(n=2)$ & $2.52 \times 10^{-3}(n=16)$ & $4.11 \times 10^{-3}(n=16)$ \\
$3.75 \times 10^{-6}(n=3)$ & $6.30 \times 10^{-4}(n=32)$ & $9.76 \times 10^{-4}(n=32)$ \\
\hline
\end{tabular}

TABLE 5: Numerical results for Example $2(b=0.25)$.

\begin{tabular}{lccc}
\hline$x$ & $y_{2}$ solution & $y_{3}$ solution & Exact solution \\
\hline 0.1 & -1.386296861 & -1.386296861 & -1.386296861 \\
0.2 & -1.386374358 & -1.386374358 & -1.386374358 \\
0.3 & -1.386901676 & -1.386901676 & -1.386901677 \\
0.4 & -1.388851090 & -1.388851090 & -1.388851090 \\
0.5 & -1.394076501 & -1.394076501 & -1.394076502 \\
0.6 & -1.405547818 & -1.405547818 & -1.405547818 \\
0.7 & -1.427453099 & -1.427453099 & -1.427453099 \\
0.8 & -1.465031592 & -1.465031601 & -1.465031602 \\
0.9 & -1.523986605 & -1.523986761 & -1.523986772 \\
1.0 & -1.609436979 & -1.609437790 & -1.609437912 \\
\hline
\end{tabular}

TABLE 6: Maximum error for Example $2(b=0.25)$.

\begin{tabular}{lcc}
\hline Present method & Method in [9] & Method in [3] \\
\hline $9.33 \times 10^{-7}(n=2)$ & $5.60 \times 10^{-4}(n=16)$ & $7.85 \times 10^{-4}(n=16)$ \\
$1.22 \times 10^{-7}(n=3)$ & $1.41 \times 10^{-4}(n=32)$ & $1.94 \times 10^{-4}(n=32)$ \\
\hline
\end{tabular}

TABLE 7: Numerical results for Example $2(b=0.75)$.

\begin{tabular}{lccc}
\hline$x$ & $y_{2}$ solution & $y_{3}$ solution & Exact solution \\
\hline 0.1 & -1.386296861 & -1.386296861 & -1.386296861 \\
0.2 & -1.386374358 & -1.386374358 & -1.386374358 \\
0.3 & -1.386901677 & -1.386901677 & -1.386901677 \\
0.4 & -1.388851090 & -1.388851090 & -1.388851090 \\
0.5 & -1.394076501 & -1.394076502 & -1.394076502 \\
0.6 & -1.405547818 & -1.405547818 & -1.405547818 \\
0.7 & -1.427453098 & -1.427453099 & -1.427453099 \\
0.8 & -1.465031590 & -1.465031600 & -1.465031602 \\
0.9 & -1.523986772 & -1.523986737 & -1.523986772 \\
1.0 & -1.609436938 & -1.609437612 & -1.609437912 \\
\hline
\end{tabular}

TABLE 8: Maximum error for Example $2(b=0.75)$.

\begin{tabular}{lcc}
\hline Present method & Method in [9] & Method in [3] \\
\hline $9.74 \times 10^{-7}(n=2)$ & $1.04 \times 10^{-3}(n=16)$ & $7.94 \times 10^{-4}(n=16)$ \\
$3.00 \times 10^{-7}(n=3)$ & $2.61 \times 10^{-4}(n=32)$ & $2.00 \times 10^{-4}(n=32)$ \\
\hline
\end{tabular}

\section{References}

[1] J. H. He, "Variational iteration method-a kind of non-linear analytical technique: some examples," International Journal of Non-Linear Mechanics, vol. 34, no. 4, pp. 699-708, 1999.

[2] J.-H. He, "Variational iteration method-some recent results and new interpretations," Journal of Computational and Applied Mathematics, vol. 207, no. 1, pp. 3-17, 2007.

[3] R. K. Pandey and A. K. Singh, "On the convergence of a finite difference method for a class of singular boundary value problems arising in physiology," Journal of Computational and Applied Mathematics, vol. 166, no. 2, pp. 553-564, 2004.

[4] J. A. Adam, "A simplified mathematical model of tumor growth," Mathematical Biosciences, vol. 81, no. 2, pp. 229-244, 1986.

[5] H. P. Greenspan, "Models for the growth of a solid tumor by diffusion," Studies in Applied Mathematics, vol. 4, pp. 317-340, 1972.

[6] S. H. Lin, "Oxygen diffusion in a spherical cell with nonlinear oxygen uptake kinetics," Journal of Theoretical Biology, vol. 60, no. 2, pp. 449-457, 1976.

[7] B. F. Gray, "The distribution of heat sources in the human head-theoretical consideration," Journal of Theoretical Biology, vol. 82, no. 3, pp. 473-476, 1980.

[8] N. S. Asaithambi and J. B. Garner, "Pointwise solution bounds for a class of singular diffusion problems in physiology," Applied Mathematics and Computation, vol. 30, no. 3, pp. 215-222, 1989.

[9] M. Abukhaled, S. A. Khuri, and A. Sayfy, "A numerical approach for solving a class of singular boundary value problems arising in physiology," International Journal of Numerical Analysis and Modeling, vol. 8, no. 2, pp. 353-363, 2011.

[10] A. M. Wazwaz, "The variational iteration method for solving nonlinear singular boundary value problems arising in various physical models," Communications in Nonlinear Science and Numerical Simulation, vol. 16, no. 10, pp. 3881-3886, 2011.

[11] A. S. V. Ravi Kanth and K. Aruna, "He's variational iteration method for treating nonlinear singular boundary value problems," Computers \& Mathematics with Applications, vol. 60, no. 3, pp. 821-829, 2010.

[12] J. He, "Variational iteration method for delay differential equations," Communications in Nonlinear Science and Numerical Simulation, vol. 2, no. 4, pp. 235-236, 1997.

[13] J.-H. He, "Variational iteration method for autonomous ordinary differential systems," Applied Mathematics and Computation, vol. 114, no. 2-3, pp. 115-123, 2000.

[14] J.-H. He, "Variational principles for some nonlinear partial differential equations with variable coefficients," Chaos, Solitons \& Fractals, vol. 19, no. 4, pp. 847-851, 2004.

[15] J.-H. He, "Some asymptotic methods for strongly nonlinear equations," International Journal of Modern Physics B, vol. 20, no. 10, pp. 1141-1199, 2006.

[16] Z. M. Odibat and S. Momani, "Application of variational iteration method to nonlinear differential equations of fractional order," International Journal of Nonlinear Sciences and Numerical Simulation, vol. 7, no. 1, pp. 27-34, 2006.

[17] S. Momani and Z. Odibat, "Numerical comparison of methods for solving linear differential equations of fractional order," Chaos, Solitons \& Fractals, vol. 31, no. 5, pp. 1248-1255, 2007.

[18] J. Lu, "Variational iteration method for solving two-point boundary value problems," Journal of Computational and Applied Mathematics, vol. 207, no. 1, pp. 92-95, 2007.

[19] J. H. He, "Asymptotic methods for solitary solutions and compactons," Abstract and Applied Analysis, vol. 2012, Article ID 916793, 130 pages, 2012.

[20] M. Abramowitz and I. A. Stegun, Handbook of Mathematical Functions, Dover, New York, NY, USA, 1972.

[21] D. Khojasteh Salkuyeh, "Convergence of the variational iteration method for solving linear systems of ODEs with constant coefficients," Computers \& Mathematics with Applications, vol. 56, no. 8, pp. 2027-2033, 2008. 


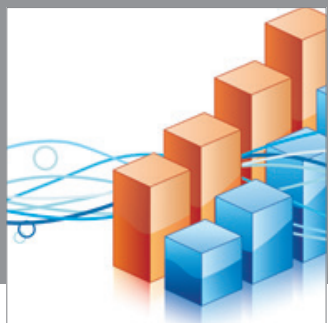

Advances in

Operations Research

mansans

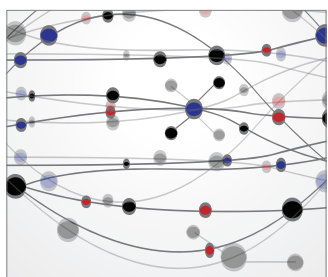

The Scientific World Journal
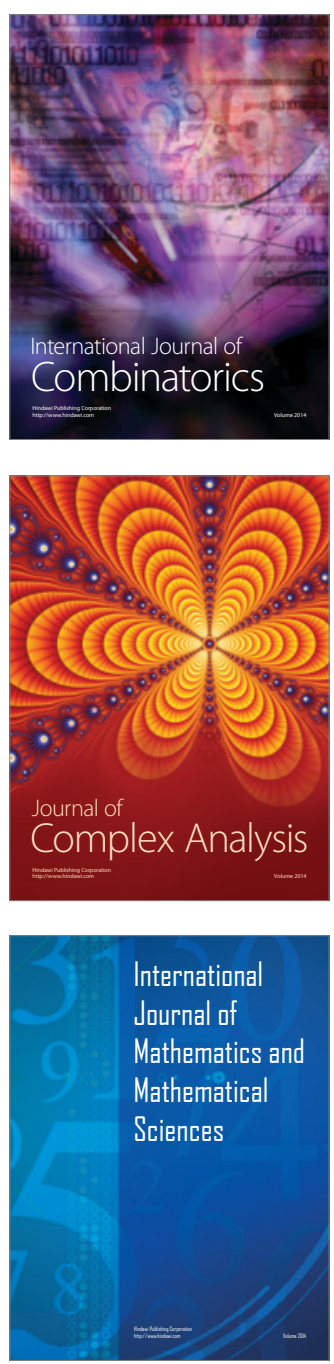
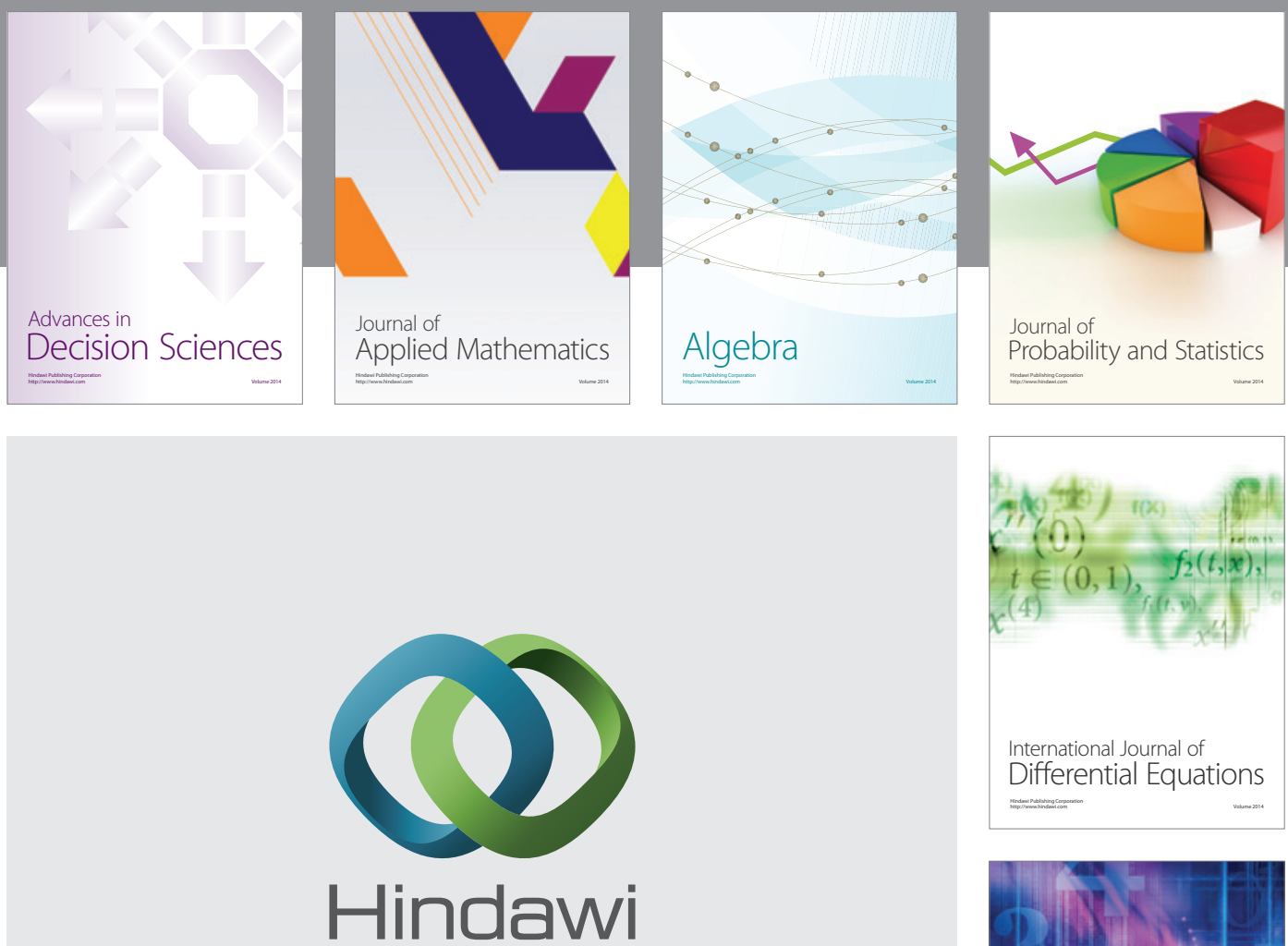

Submit your manuscripts at http://www.hindawi.com
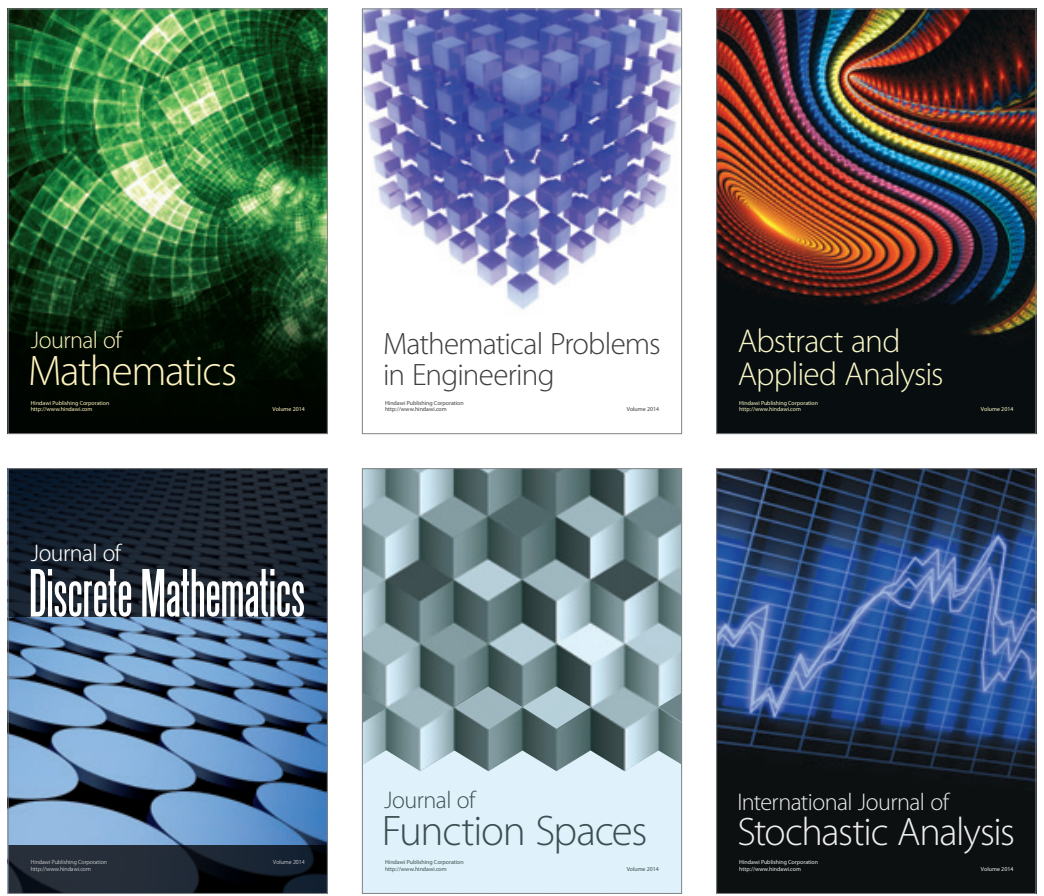

Journal of

Function Spaces

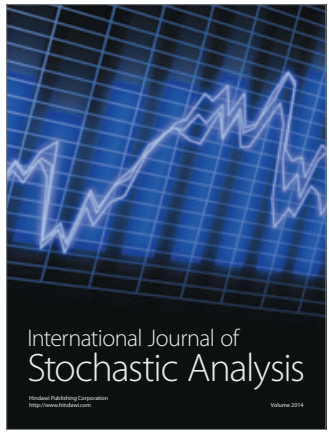

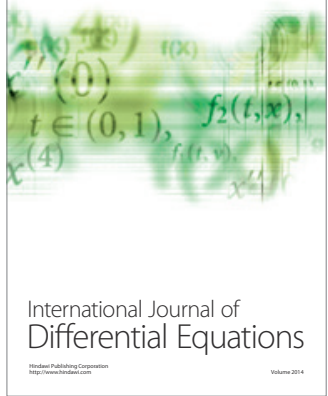
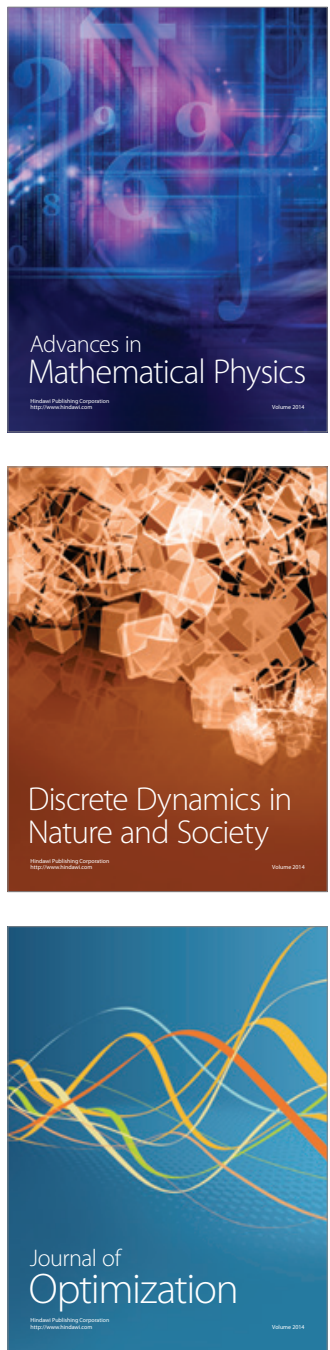